\title{
Experimental and numerical investigation of real world dimpled heat exchanger
}

\author{
S. Preibisch \& M. H. Buschmann \\ Institute of Air Handling and Refrigeration Dresden, \\ Department of Air Handling Technology, Germany
}

\begin{abstract}
This study is motivated by the observation that recent investigations of dimpled surfaces rarely go beyond single plates or channels. Here, we explore a real world cross flow heat exchanger $\left(400 \times 400 \times 100 \mathrm{~mm}^{3}\right)$. It is found that in any dimpled configuration the exchanged heat is larger than in the reference case (smooth walls). The enhancement of the specific heat transfer per unit area can exceed $40 \%$. Reasonable agreement of global parameters with numerical simulations (FLUENT) is obtained when the whole heat exchanger considering each individual structure is modelled. Based on that finding we analyse the numerical results in order to obtain information on local flow situation and in order to find physical explanations for the different enhancement found in different geometrical configurations.
\end{abstract}

Keywords: heat exchangers, dimples, experiment, numerical simulation.

\section{Introduction}

Heat exchangers play a central role in process engineering, air conditioning heating and many other technical applications. Analysing the international literature, we see great progress being made in developing new and advanced technologies to improve traditional heat exchangers like cross- and counter-flow heat exchangers (see e.g. Gad-el-Hak [1]). However, the majority of these investigations are restricted to rather less academic subjects. In reality, a heat exchanger is much more complex than the often examined single plates and passages.

In this study we look at a complete cross-flow heat exchanger. Our general strategy is to combine experimental investigations of global parameters like 
pressure losses and heat transfer coefficients with numerical results. The main goal of the study is to explain the former parameters which are most important for heat exchanger operators employing detailed information obtained from simulation.

\section{Dimpled surfaces}

During the last two decades or so, dimpled surfaces have become a new option for the improvement of heat transfer by a comparably small increase of pressure loss. Experimentally investigated dimples are mostly shallow, circular cavities. Their diameter to depth is comparable large with 4.3-5.0 (Park et al. [2]) to 20 (Lienhart et al. [3]). Such structures are difficult to insert both-sided into heat exchanger sheets. It is more realistic to deform sheets in such a way that valleys appear on one side and crests on the opposite side. In general building heat exchanges employing such sheets two different geometrical configurations are possible. Either channels having crests on both sides alter with channels having valleys on both sides (Fig. 1(a)) or all channels are equal (Fig. 1(b)). In the latter case the channels have crests on one side and valleys on the opposite side. Those geometries also allow anticipating an increase of heat transfer (Hwang et al. [4]). However, compared to surfaces which have really shallow dimples like the structures employed by Lienhart et al. [3], an increased of pressure loss must be expected especially in crested channels.

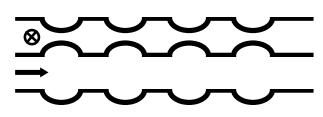

(a)

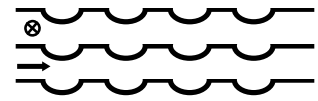

Figure 1: Geometrical configurations (arrows indicate possible flow direction).

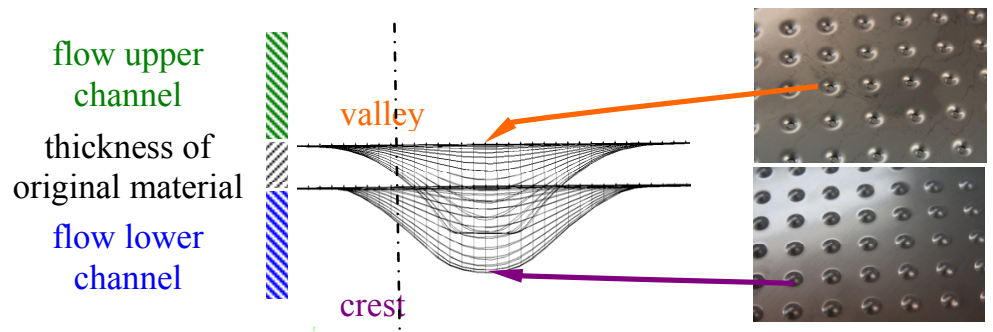

Figure 2: Employed heat exchanger sheets (right) and 3d-surface scan (centre). (Colour version online.)

In the present study we use cold-worked sheets manufactured from plane steal sheets $\left(400 \times 400 \mathrm{~mm}^{2}\right)$ originally $1 \mathrm{~mm}$ thick. Dimple valleys were machined by metal spinning employing a cylindrical embossing die. Figure 2 shows a 3D- 
surface scan of one single structure. The averaged depth was $2 \mathrm{~mm}$ at most and the outer diameter about $9 \mathrm{~mm}$. The structures were placed equidistantly in streamwise and cross-flow direction with a distance of about $25 \mathrm{~mm}$. Note that the structures do not show any sharp edges which is basically caused by the manufacturing technology. With a diameter to depth ratio of 4.5 it is close to [2]. The increase of surface area due to dimpling is about $9.6 \%$.

\section{Experimental setup and measurement regime}

The test facility employed basically consists of two small intersecting wind tunnels (Fig. 3). The cross-flow heat exchanger is placed directly in the intersection point. It has five passages for hot and five passages for cold airstream. Each channel has a clear height of $9 \mathrm{~mm}$. As reference case a crossflow heat exchanger with smooth sheets of $1 \mathrm{~mm}$ thickness is employed.

The hot airstream can be heated up to $120^{\circ} \mathrm{C}$ and is guided in a completely insulated closed loop. Comparable to any wind tunnel, both airstreams before entering the heat exchanger are conditioned by honeycombs, several grids with downstream degreasing mesh size, a settling chamber and finally a nozzle (contraction 1:2.5). To ensure a homogeneous temperature profile of the hot stream, additional mixers are built-in after the heaters.

Temperature and pressure are measured shortly before the entrances and shortly after the exits of the heat exchanger. Temperature is averaged from four thermocouples positioned equidistantly across inlet and outlet cross-sections. Additionally, air humidity is measured in order to calculate air properties accurately. Flow rates are determined using standard orifices. However, the construction of the test rig allows only entrance lengths upstream of these orifices which are not conform to standards. Therefore the flow rate

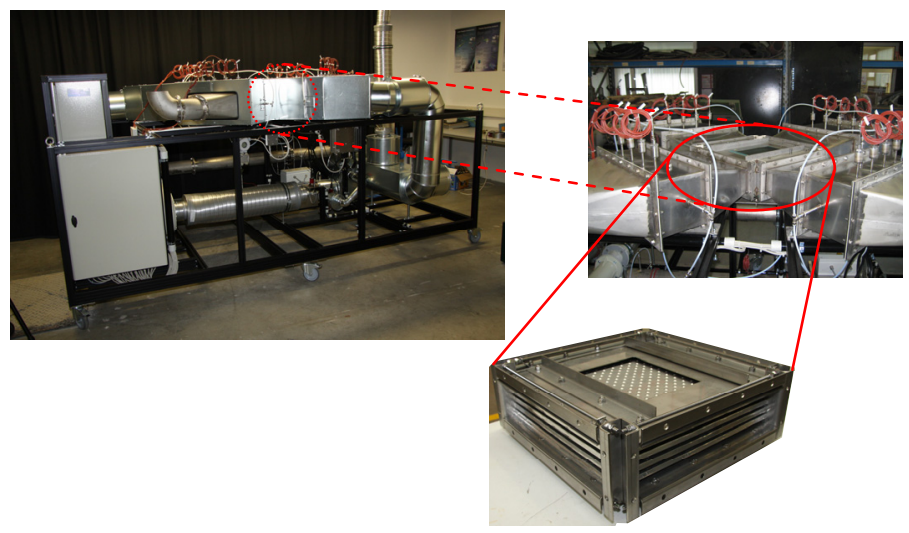

Figure 3: Test facility; top left - overall view, top right - heat exchanger inside non-insulated test facility and below - heat exchanger with dimpled sheets. (Opening on top of heat exchanger is for laser optical investigations which are not discussed here.) (Colour version online.) 
measurements have been calibrated against well defined flow rates provided by an external blower. Data acquisition is fully automated based on LABVIEW.

For each investigated geometrical configuration (see Tab. 1) including the reference case the same measurement regime is accomplished. The cold airstream is varied in seven equidistant steps between $120 \mathrm{~m}^{3} / \mathrm{h}$ and $460 \mathrm{~m}^{3} / \mathrm{h}$ (2.600 $\left.\delta \operatorname{Re}_{c} \delta 9.600\right)$. This is done for the three flow rates $240,290,345 \mathrm{~m}^{3} / \mathrm{h}$ of the hot air stream $\left(R e_{h} \approx 3000,3600,4300\right)$. In any case the hydraulic diameter of a single passage, the bulk velocity in the passages and an average kinematic viscosity is employed to build the Reynolds numbers.

Table 1: Geometrical configurations.

\begin{tabular}{|c|c|c|}
\hline Name & Specification & $\begin{array}{c}\text { Symbols in Figs. } \\
R e_{h}=3000,3600 \\
4300\end{array}$ \\
\hline $\begin{array}{l}\text { reference } \\
\text { case }\end{array}$ & $\begin{array}{l}\text { smooth sheets no } \\
\text { structures }\end{array}$ & $\begin{array}{l}\text { experiment } \Delta \square \\
\text { simulation } \Delta \square \diamond\end{array}$ \\
\hline series 1 & $\begin{array}{c}\text { geometry according to } \\
\text { Fig. 1a }\end{array}$ & $\begin{array}{l}\text { experiment } \Delta \square \diamond \\
\text { simulation } \Delta \square \diamond\end{array}$ \\
\hline series 2 & $\begin{array}{c}\text { geometry according to } \\
\text { Fig. } 1 \mathrm{~b}\end{array}$ & $\begin{array}{l}\text { experiment } \Delta \square \\
\text { simulation } \Delta \square \diamond\end{array}$ \\
\hline
\end{tabular}

On average the relative experimental error obtained with the test facility is $\pm 4 \%$ for Reynolds number $\pm 5 \%$ for heat flux and $\pm 7 \%$ for pressure loss coefficient. The thermal performance parameter $T_{3}$ has a relative error of $\pm 12 \%$ (for definition see section 5).

\section{Numerical investigation}

Numerical simulations were carried out on a PC-cluster of six cores employing FLUENT 12. A blending of $\mathrm{k} \omega$-model for flow regions very close to walls and

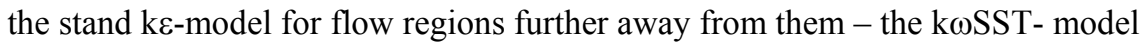
- was employed for all flow configurations. All simulations were conducted for compressible flow. The heat exchangers were completely three-dimensionally resolved including all individual structures (valleys/crests). Additionally buffer regions were placed after the outlets of the heat exchanger to ensure numerical stability inside the flow domain of interest. For the reference case a total number of grid points of about $8 \times 10^{6}$ and for all other cases of about $12-14 \times 10^{6}$ was implemented. Due to the simpler geometry, the reference case (smooth walls) could be constituted only from hexahedrons. For the more complex geometry of dimpled cases combinations of polyhedrons and tetrahedrons were employed to meet the surface in detail. The dimpled surfaces of the cold-worked sheets actually employed in the experiments were scanned (see Fig. 2) and 
implemented as IGEST-files. Grid independency of the results was checked by several test runs with different mesh sizes. Stop criteria were set at $10^{-8}$ for the energy transport equation, $10^{-3}$ for the continuity and $10^{-4}$ for all other equations. CPU-time per heat exchanger realization was about $30 \mathrm{~h}$.

\section{Data processing}

The aim of our study is to create design rules for the practical application of dimpled surfaces in future heat exchangers. Therefore it is important to characterize the different geometrical configurations by their pressure losses written as non-dimensional pressure loss coefficient.

$$
\zeta(R e)=\Delta p / \frac{\rho}{2} u_{b}^{2}
$$

A direct experimental determination of local Nusselt numbers inside the experimental investigated heat exchanger is not possible. It is also not possible to decompose the global heat transfer coefficient $k$ into two global Nusselt numbers for the simple reason that the Nusselt number is different on the different sides of one heat exchanger sheet. This is due to the different geometries (valleys on one side and crests on the other side) and possible Prandtl number effects. Therefore the global $k$-value is considered the central parameter in characterizing the different geometries from a thermodynamical point of view. It is calculated applying the Betriebscharakteristiken-method as described by Bosnjakovic and Knoche [5]. (The method is based on the idea that any heat exchanger can be described depending on the non-dimensional numbers $B_{I}=k A / C_{c}$ and $B_{2}=C_{c} / C_{h}$ were $C$ denotes cold and hot heat capacity flux.)

There are several approaches and parameters exist which evaluate and compare heat exchangers with varying designs (see e.g. Sahiti et al. [6]). Here, we follow the notion of the so-called thermal performance parameters proposed by for example Hwang et al. [4] and Burgess and Ligrani [7]. Usually these parameters are built based on time and space averaged Nusselt numbers. As explained above such numbers cannot be derived experimentally in our case. Therefore we design the following thermal performance parameter:

$$
T_{i}=\left(\frac{k}{k_{0}}\right)^{m}\left[\left(\frac{\zeta_{0 c}}{\zeta_{c}}\right)^{n}\left(\frac{\zeta_{0 h}}{\zeta_{h}}\right)^{n}\right]^{\frac{1}{2}} \quad T_{i}=\left(\frac{k}{k_{0}}\right)^{m}\left(\frac{\zeta_{0}}{\zeta}\right)^{n}
$$

The subscripts $c$ and $h$ stand for cold and hot channel and 0 denotes the reference case. The powers $m$ and $n$ can be chosen freely. Here they are set as $m=1$ and $n=1 / 3$ in accordance with equivalent thermal performance parameters in $[4,7]$. Hence $T_{i}$ will be called $T_{3}$ in the following. Note that eq. (2a) considers the pressure loss coefficients of both passages because they may have different $\zeta$-values due to the different geometries and due to different Reynolds numbers. Only in case both passages have the same geometry and the same Reynolds number (2a) reduces to (2b). In case $T_{3}$ takes values larger than unity, the dimpled heat exchanger performs better than the reference case in the sense that 
the inevitable increase of pressure loss is compensated by the desired increase of heat transfer.

\section{Results}

In a first step we look at the pressure losses caused by the different geometrical configurations (Fig. 4). Obviously the passages with smooth walls (reference case) have the lowest $\zeta$-values. However, passages with both sides structured with valleys show only a very small increase. The reason is that the flow runs through the core regions of these passages without meeting any obstacles on its way. Therefore the form drag of such passages is hardly increased. The contrary happens in passages having crests on both sides. Here, the pressure loss is roughly doubled due to a massive increase in form drag. Note that the crests (like the valleys) are positioned vis-á-vis and that the wall normal clearance between the crest tops is about $4 \mathrm{~mm}$. The clearance of the undeformed parts of these passages is still $9 \mathrm{~mm}$, like in the reference case. Passages having one side dimpled (valleys) and one side crested are found between these two extremes discussed earlier. The clearance of these passages is the same nearly everywhere. However, these passages meander up and down which of course increases drag.
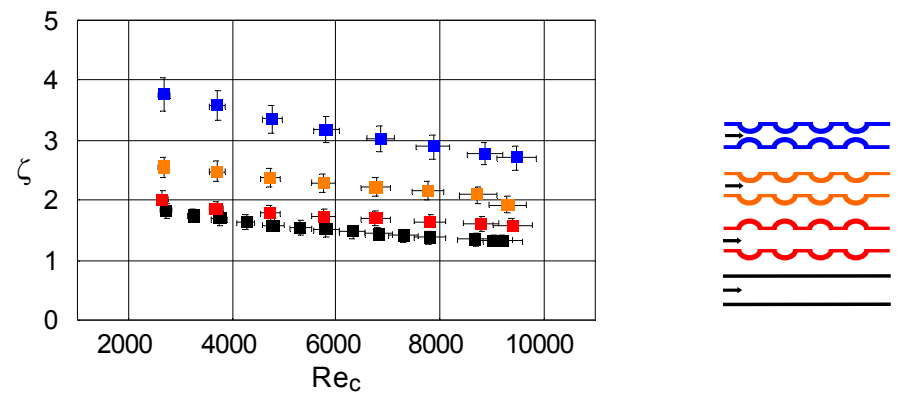

Figure 4: Pressure loss of geometrically different passages. Sketch indicates the different geometries. Arrows show flow direction. (Colour version online.)

Figure 5 compares experimental with numerical results for global heat transfer coefficients. Plot 5a shows that the $k$-value is significantly increased for all structured cases. However, the increase changes with $R e_{c}$ and may reach values up to $40 \%$ for $R e_{c}$ larger than 8000 . Series 1 and 2 show a nearly equal enhancement for $R e_{c}$ larger than 7000. However, in a $R e_{c}$-range of about 30006000 , series 2 performs better. We will come back to this point later on. For $R e_{c}$ below 3000, nearly no improvement is found compared with the reference case.

The reference case (Fig. 5(b)) is simulated in near perfect agreement with experiments. The simulations of series 1 and 2 (Fig. 5(c), (d)) show reasonable accordance with experiments. Differences are between -8 and $16 \%$ depending on the Reynolds number and on geometrical configuration.

Figures 7 and 8 compile information obtained from numerical simulation for a Reynolds number combination of $R e_{c}=4400$ and $R e_{h}=3700$. This specific 

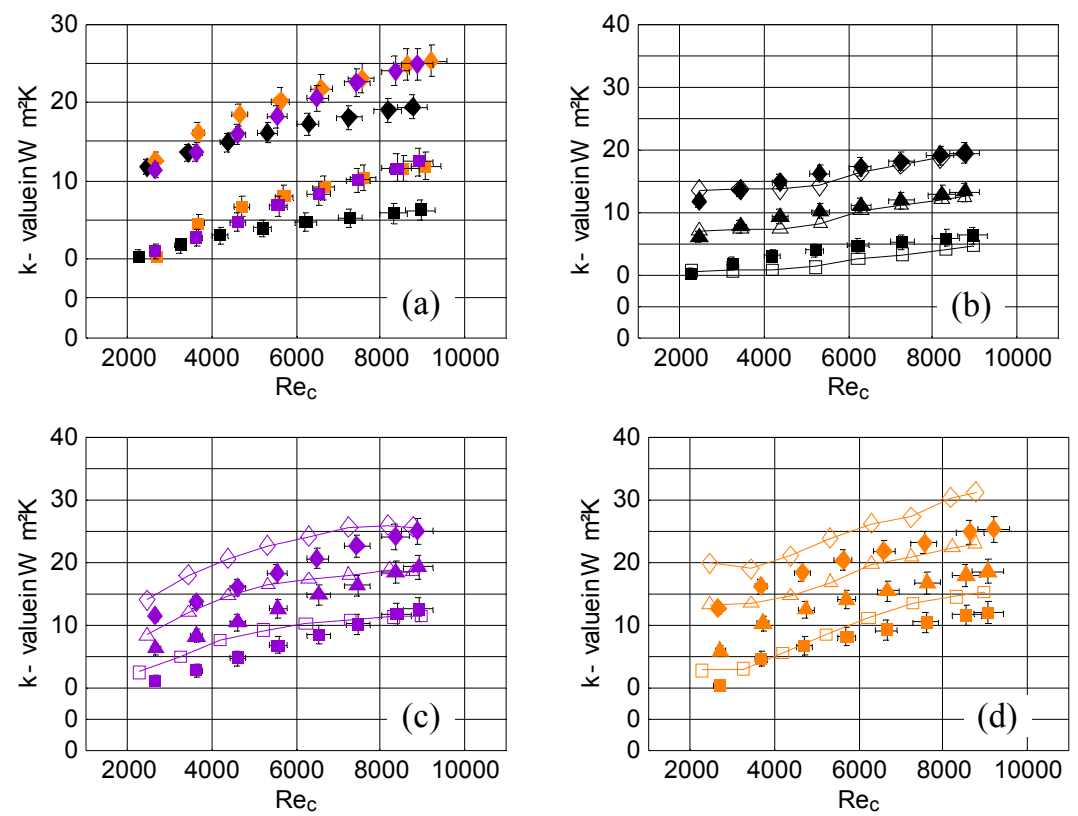

Figure 5: Heat transfer coefficients. Plot (a) compares experimental results for two different $R e_{h}$ in the hot passage and (b-d) show comparison with numerical results. The $k$-values are shifted by $\Delta k=5 \mathrm{~W} / \mathrm{m}^{2} \mathrm{~K}$ $\left(R e_{h}=3600\right)$ and by $\Delta k=10 \mathrm{~W} / \mathrm{m}^{2} \mathrm{~K}\left(R e_{h}=4300\right)$. Curves are added for visual aid only. (Colour version online.)

case was chosen to identify possible reasons for the experimentally found differences between series 1 and 2 mentioned earlier. The large show plots always the spatial distribution of the mean temperature in the central plain positioned equidistantly between the non-deformed parts of the sheets building one passage. In all cases the typical diagonal temperature distribution of crossflow heat exchangers are found. Additional comb-shaped patterns are identified which are caused by the streamwise oriented alignments of structures. Because the thermodynamical and the fluid mechanical entrance conditions are equal in all simulations differences can only be caused by the different geometries. To highlight these differences in more detail, the mean temperature and the streamwise and the wallnormal velocity components are plotted in the central longitudinal section.

To illustrate the enhancement of the wallnormal velocity component with respect to the structures this velocity component is also shown for the reference case with smooth walls (Fig. 6). A comparison shows two things. First, the wallnormal velocity component is about ten times larger in the structured cases than in the unstructured case and second; the typical entrance length expected in straight channels (indicated by the distinctive blue and red regions in Fig. 6 colour as seen online) does not exist in any of the structured passages. In general 
it can be stated that the investigated configurations do not show any entrance effects. This is different to e. $g$. tube bundle heat exchanges where the first tube rows show such effects.

Figure 6: Wall normal velocity component of smooth heat exchanger 0.04 $\mathrm{m} / \mathrm{s} \leq \mathrm{w} \leq 0.04 \mathrm{~m} / \mathrm{s}$. (Colour version online.)

Comparing the streamwise velocity component, it becomes clear that in symmetric flow situations (valley-valley channel and crest-crest channel Fig. 7), the flow forms a kind of jet running through the undisturbed core region of the channel. This is especially true for the valley-valley channels. The overall flow situation indicated here by the wallnormal velocity component is more or less symmetric to the central plain of the passage. The wallnormal velocity component itself has a value of nearly zero in this plain. This is different in a valley-crest passage (Fig. 8). Due to the snake-like geometry, the flow meanders weakly around the central plain here. Therefore the wallnormal velocity component shows a completely different behaviour. Not only the regions of significant wallnormal flow short before and short after the structures are larger but their intensity is also higher than in the symmetric case (compare e. g. uppermost plots in Figs. 7 and 8). Fluid is even transported across the central plain which leads to an improved wallnormal mixing. This in turn makes the comb-shaped pattern of the mean temperature more blurred (compare areas downstream of the broken lines in Figs. 7 and 8). The longitudinal section of mean temperature shows an increasing asymmetry along the streamwise direction. This altogether explains why for $3000 \delta \operatorname{Re}_{c} \delta 5000$ series 2 the experimentally $k$-value is about $10 \%$ higher than for series 1 .

Experimentally obtained thermal performance parameters $T_{3}$ (eq. 2) are compiled in Fig. 9. This parameter increases when the $k$-value of a structured heat exchanger is larger than the equivalent value of the reference case or when the pressure loss coefficients are smaller than the equivalent values of the reference case. The latter can hardly be achieved as shown in Fig. 4. The consequence is that the heat transfer represented by the $k$-value has to be enhanced above average to obtain $T_{3}$-values larger than unity. Fig. 9 shows that this is the case for series 1 for $R e_{c}$ larger than about 6000 and for series 2 for $R e_{c}$ larger than about 3000. There are two facts which make series 2 the best. First, the pressure loss in all passages is significantly lower here than in the crest-crest passage of series 1 . This is obviously also not compensated by the very low pressure loss in the valley-valley passages of series 1 . The second point is the higher $k$-value of series 2 in the $R e_{c}$-range between 3000 and 6000 . However, for $R e_{c}$ larger than about 8000 this bonus of series 2 is depleted and both geometrical configurations show similar thermal performance parameters. Note that all thermal performance parameters shown here, are significantly smaller than those built with Nusselt numbers and cannot be compared with such parameters directly. 

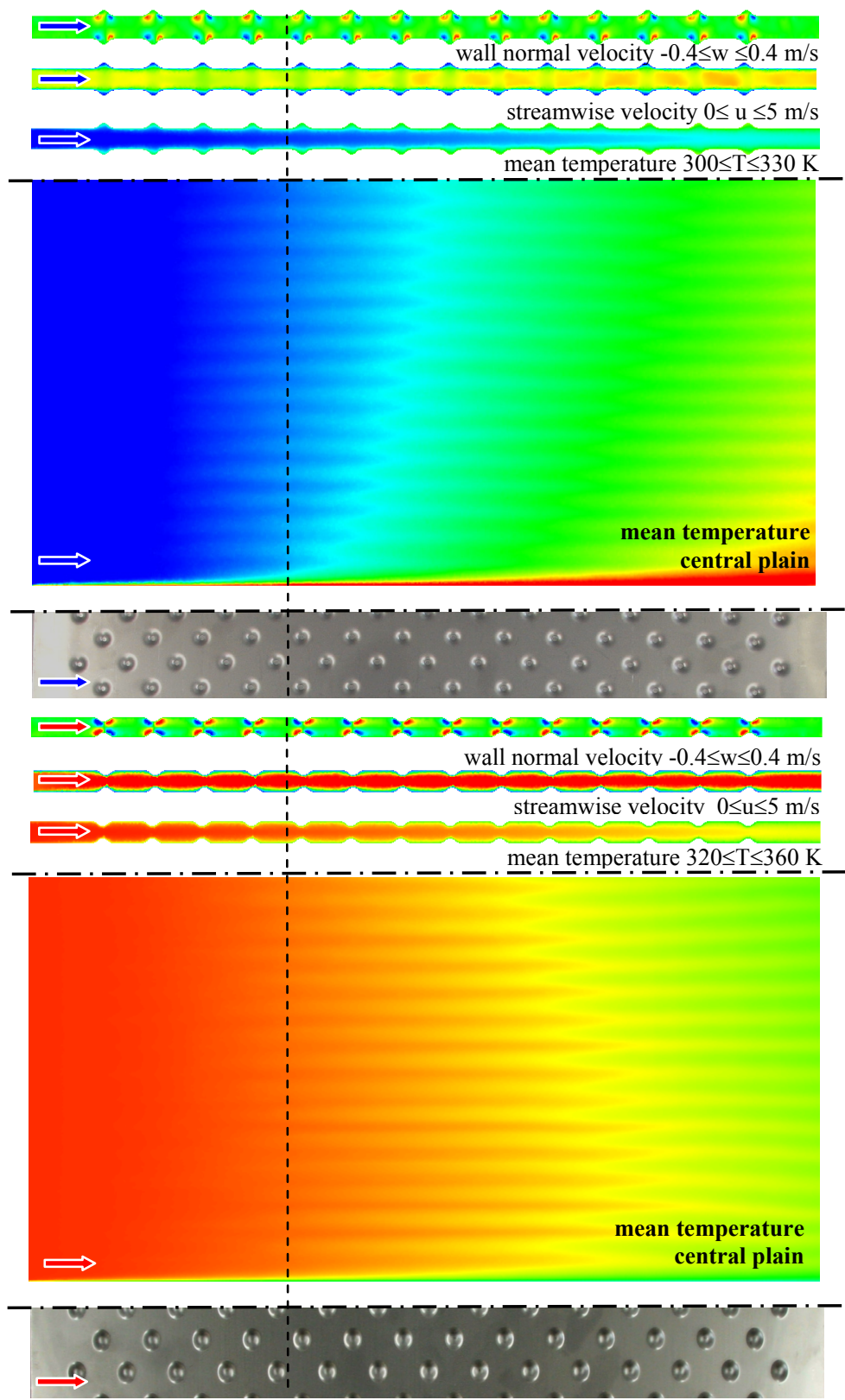

Figure 7: Cold valley-valley channel (above) and hot crest-crest channel (below) Sheets show structure on both channel sides. Broken lines indicate one row of structures. (Colour version online.) 
32 Advanced Computational Methods and Experiments in Heat Transfer XI
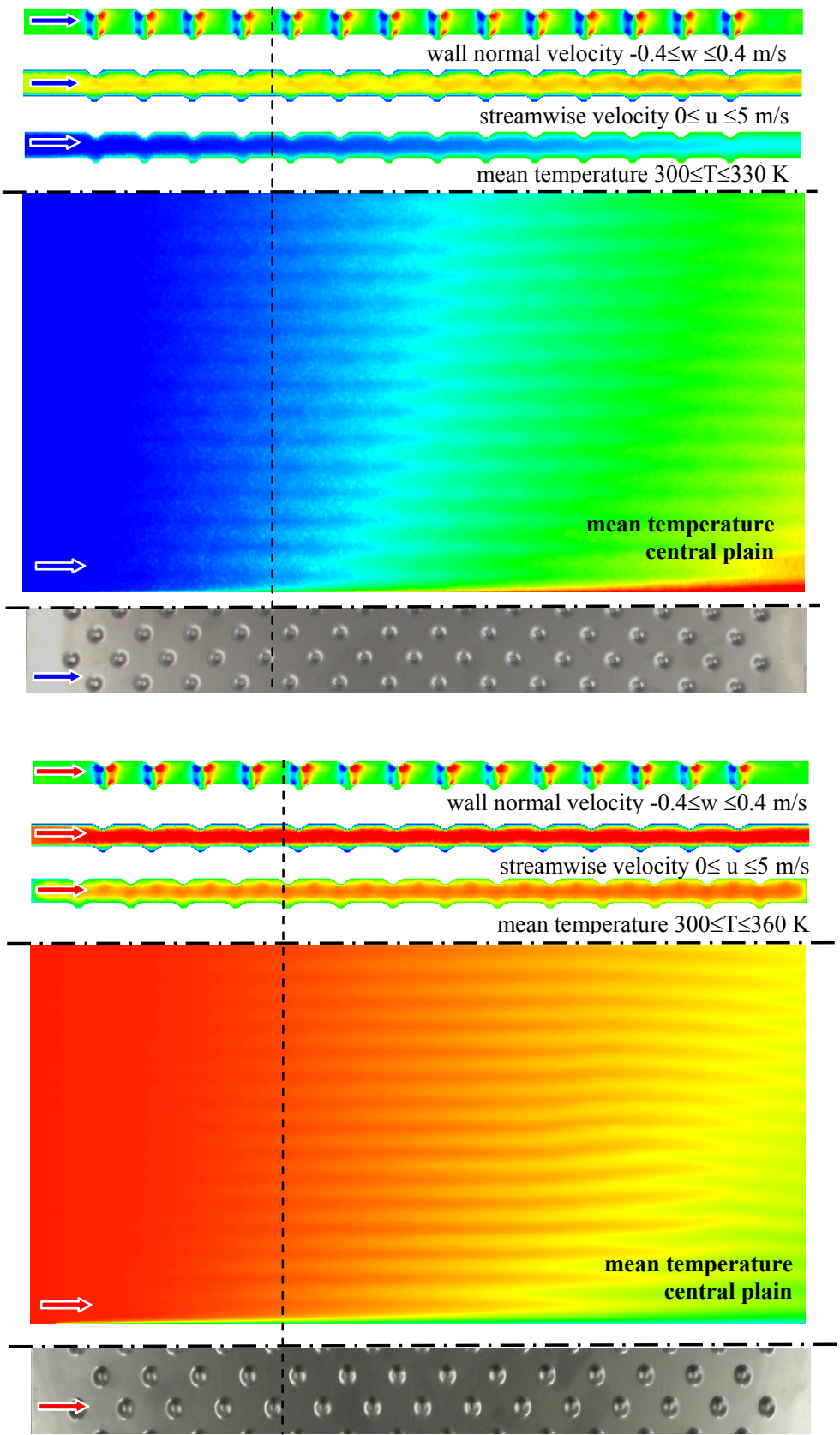

Figure 8: Cold valley-crest channel (above) and hot valley-crest channel (below) Sheets show structure on lower channel sides only. Broken lines indicate one row of structures. (Colour version online.) 


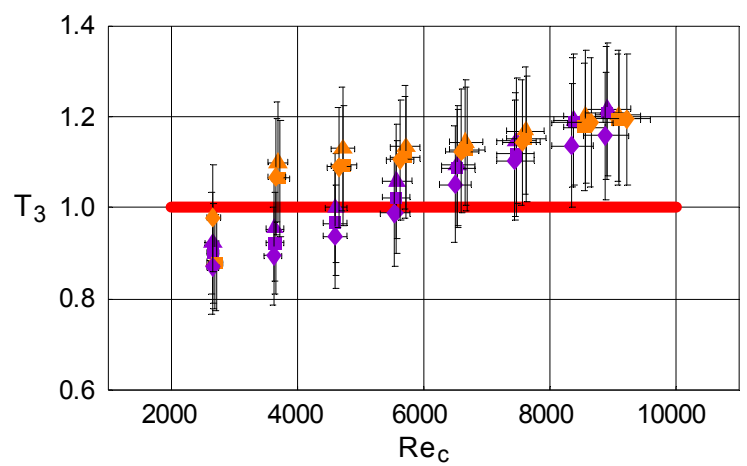

Figure 9: Experimentally obtained thermal performance parameters for series 1 and series 2 . Red bold line indicates $T_{3}=1$. (Colour version online.)
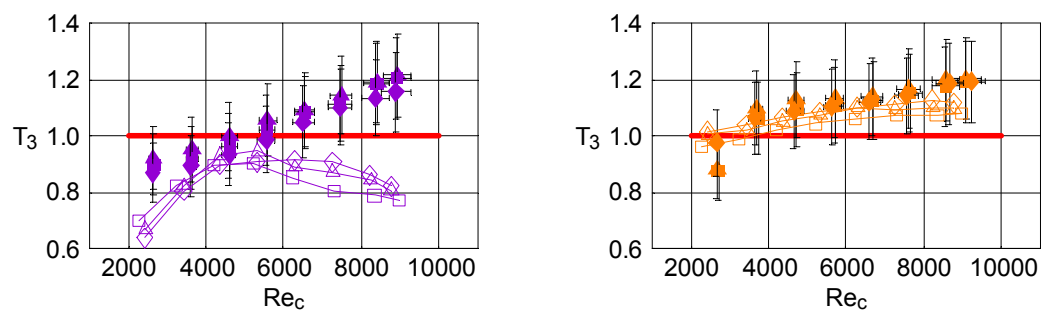

Figure 10: Comparison of experimentally and numerically obtained thermal performance parameters for series 1 (left) and series 2 (right). Red bold line indicates $T_{3}=1$. Curves are added for visual aid only. (Colour version online.)

Figure 10 compares thermal performance parameters obtained experimentally and numerically. The agreement for series 2 is reasonable. All numerical results are found within the error bars of the experimental values. The general trend is met both qualitatively and quantitatively. Unfortunately this is not the case for series 1 . For $R e_{c}$ between 3000 and 5000 the numerical results come close to the experiments. For higher Reynolds numbers a significant difference between both is found. We can trace this discrepancy back to erroneous simulated pressure loss of the crest-crest passage. The vigorous changes in geometry cause a complex flow field with strong velocity gradients which make simulation more challenging here than in the other passages. Obviously an even higher spatial resolution than employed so far has to be implemented to capture of flow features properly.

\section{Conclusions}

Experimental and numerical results for dimpled cross-flow heat exchangers are presented. The differences of two geometrical configurations found 
experimentally are explained using the numerical simulations. A preference with respect to one of the geometries is given.

The combination of experimentally obtained global parameters ( $k$-value, pressure losses) with numerically predicted local parameters (temperature, velocity etc.) seems to be a promising engineering strategy for the investigation of advanced heat exchanger designs. However, the effort is definitively shifted from traditional experiments to numerics. Specific effort is required to simulate a real world heat exchanger. It is not sufficient to restrict the simulations to only part of the apparatus. Instead, the whole heat exchanger needs to be considered. Additionally, the actually manufactured geometry has to be measured (scanned) in order to properly represent this geometry in the simulations. The latter is also most important to obtain the effective heat transfer area. The consequence is that not only sufficient experimental and computational power is needed. Sophisticated knowledge concerning the simulation of real world heat exchangers is required as well.

\section{Acknowledgements}

The present work was done under grant EURONORM VF071025. Funding for the computer instrumentation used in this study was provided by SAB grant ER 2191-2006. The authors also wish to thank Stefan Pohl and Christian Friebe for supporting us with the experimental work.

\section{References}

[1] Gad-el-Hak, M. Flow Control: Passive, Active, and Reactive Flow Management. Cambridge University Press, 2000, reprinted 2006.

[2] Park, D., Silva, C., Marotta, E. \& Fletcher, L. Study of laminar forced convention heat transfer for dimpled heat sinks. Journal Thermophysics, 22, pp. 262-270, 2008.

[3] Lienhart, H., Breuer, M. \& Köksoy, C. Drag reduction by dimples? - A complementary experimental / numerical investigation. $5^{\text {th }}$ International Symposium on Turbulence and Shear Flow Phenomena, Munich, 2007,

[4] Hwang, S. D., Kwon, H. G. \& Cho, H. H. Heat transfer with dimple/protrusion arrays in a rectangular duct with a low Reynolds number range. Journal of Heat and Fluid Flow, 29, pp. 916-926, 2008.

[5] Bosnjakovic, F. \& Knoche, K. F. Technische Thermodynamik. Dr. Dietrich Steinkopff Verlag, GmbH \& Co. KG Darmstadt, 1998.

[6] Sahiti, N., Durst, F. \& Dewan, A. Strategy for selection of elements for heat transfer enhancement, International Journal of Heat and Mass Transfer, 49, pp. 3392-3400, 2006.

[7] Burgess, N. K. \& Ligrani, P. M. Effects of dimple depth on channel Nusselt numbers and friction factors. Journal Heat Transfer, 127, pp. 839-847, 2005. 